\title{
The Capturing Robot with Super High Acceleration
}

\author{
Makoto Kaneko ${ }^{1}$, Reika Takenaka ${ }^{1}$, and Masatoshi Ishikawa ${ }^{2}$ \\ ${ }^{1}$ Graduate School of Engineering, Hiroshima University, Higashi-Hiroshima, \\ Japan \\ 2 Graduate School of Engineering, The University of Tokyo, Bunkyo-ku, Tokyo, \\ Japan
}

\begin{abstract}
This paper discusses the design of capturing system with an extremely high response. The key for achieving a quick response is the Arm/Gripper Coupling Mechanism (AGCM), where the potential energy initially accumulated in the arm is transferred to the kinetic energy of the arm and, continuously, to the kinetic energy for closing the gripper at the capturing point without any time lag. The experimental results show the maximum acceleration of $91 \mathrm{G}$, and the total capturing time of $0.03 \mathrm{sec}$. Capturing a dropping ball is the highlight of the experiments.
\end{abstract}

\section{Introduction}

We are now taking part in the CREST [1] project where we are exploring that how a fast vision system, such as 1ms-vision [3], [4] changes the robotic world with a combination of a high-speed robot as shown in Fig.1. For executing the project, conventional robots are generally not quick enough for capturing a moving object. Knowing of the limitation of response of conventional robots, we begin by designing a new robot system, especially with focusing on the actuation system capable of capturing a moving object in the air. For doing it, there are two key issues to be considered; (1) For the arm, how to achieve a quick motion for approaching the target object and (2) for the gripper, how to achieve a quick motion for closing it without any time lag after the arm motion. While there have been various robots with extremely high responses, in our knowledge, the Humming Bird [5] is keeping the world record with the maximum acceleration of 50G. We believe that the maximum acceleration is a good index for evaluating how quickly the robot can respond. In order to drastically increase the maximum acceleration of arm, it is necessary to fully reconsider the actuation system itself, leaving from magnetic based actuators, such as DC or AC servo motor. One good candidate is to utilize an accumulated energy, such as pneumatic or spring energy [12]-[15]. As for (1), we increase the acceleration by using the spring energy and by decreasing the weight of both arm and gripper. As for (2), we propose a new design where instead of installing actuator individually for both arm and gripper, the single energy source is continuously transmitted from the arm to the gripper through a mechanically coupled mechanism. An 


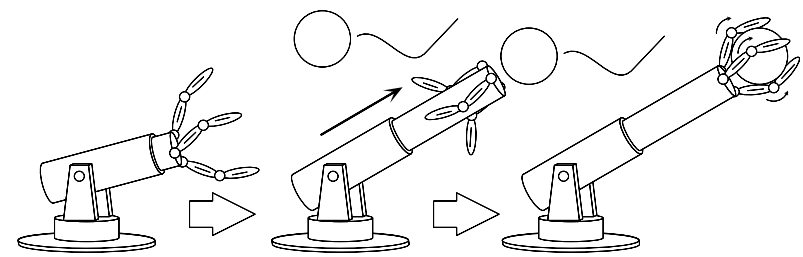

$\begin{array}{lll}\text { (a)Initial State } & \text { (b)Approach Phase } & \text { (c)Grasp Phase }\end{array}$

Fig. 1. An Image of Capturing System

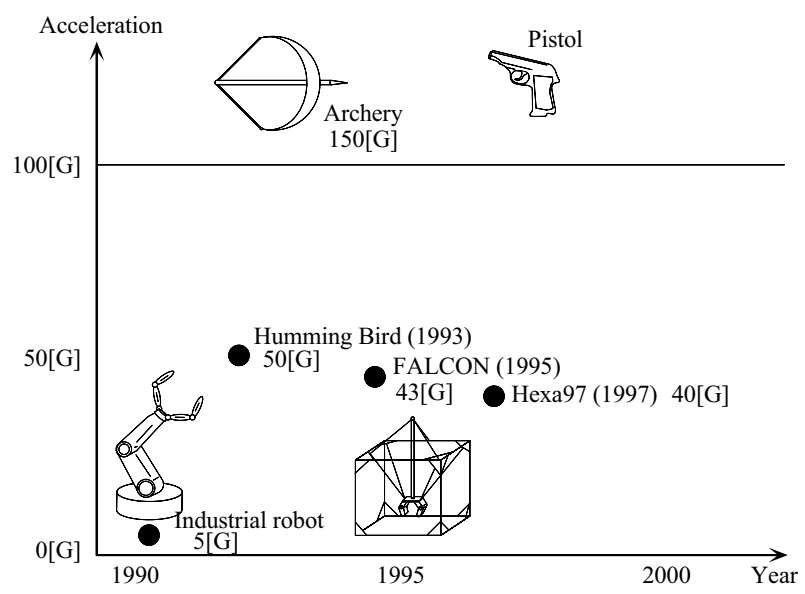

Fig. 2. Map of the Acceleration in Conventional Robot

important note in mechanical design is to consider an effective utilization of such a single energy source from arm motion to gripper motion without any time delay and without overlapping both motions. In order to give a mechanical solution for such a requirement, we utilize the Arm/Gripper Coupling Mechanism (AGCM)[2] composed of wire, stopper, and transmission plate, where all mechanical components have negligibly small mass. The AGCM is the key for effectively exchanging energy from the arm to the gripper, without the time lag and the overlapping between the arm and the gripper motions.

Based on these ideas, we design and develop the planar type capturing system composed of DC servo-motors for compressing the spring and adjusting the capturing point, a laser sensor for measuring the distance up to the object, an electromagnet for keeping spring energy. The developed capturing system can operate in two modes; normal and high-speed modes. The experimental results in high-speed mode show the maximum acceleration of $91 \mathrm{G}$ corresponding to almost two times larger than the world record, and the total capturing time of $0.03 \mathrm{sec}$. Experiments on capturing a ball under natural dropping from the height of $20 \mathrm{~cm}$ are also demonstrated. 


\section{Conventional Works}

For robot manipulators, there are two mechanical configurations: The serial link manipulator where each link is serially connected from the base to the end-effector through an actuator at each joint, and the parallel link manipulator where the base and the end-effector are connected by link mechanism arranged in parallel. Generally, a parallel link manipulator can react even faster than a serial arm, because we can greatly reduce the mass of moving part by mounting all actuators at the base. We can also classify robot manipulators according to the power transmission mechanism. While there are a number of industrial robots, most of them include the gear train to produce a sufficiently large force at the end point. This is why their accelerations are at most 5G, as shown in Fig.2. A direct drive robot where each motor shaft is directly connected to link can move even quicker than a robot with the gear train. The Barrett Arm [6] with the top speed of $5 \mathrm{~m} / \mathrm{s}$ is categorized into semi-direct drive robots. It is constructed by the combination with powerful motors at the base and tendon drive system without any gear train. The actuator speed is slightly reduced by implementing different size of pulleys between the drive and the output shafts. This design enables us to achieve a high-speed and good controllability with moderate size of actuators.

As for grasping a moving object, Allen and others have demonstrated the automated tracking and grasping of a moving object with a robotic hand [7]. They used a moving model train as an object and therefore, the grasping strategy should not be as difficult as that for capturing an object moving in the air. By using the prototype model of the Barrett Arm [8], Hong and Slotine [9] have succeeded in catching a ball under hand tosses from random locations approximately $1.5-2.5 \mathrm{~m}$ distance from the base of the arm. As far as we know, this is the first experiment succeeding in catching a moving object in the air by a robot. However, since it takes approximately $0.5 \mathrm{sec}$ for completing the whole catching motion, each toss should be strongly limited in slow speed. A robot capable of quick action is, of course, desirable from the viewpoint of accurately approaching and catching an object moving fast. In order to realize a quick motion, Kawamura and others [10] have designed an ultra high-speed robot (FALCON) by combining wire transmission with parallel mechanism, and they achieved the maximum acceleration of 45G. Uchiyama and others [11] have succeeded in attaining $40 \mathrm{G}$ by utilizing direct drive actuators with parallel mechanism. These maximum accelerations, however, suggest a potential limitation for using electromagnetic based actuators, such as DC or AC motor. On the other hand, an accumulated energy, such as pneumatic or spring one is often utilized especially in legged machines capable of jumping or hopping. Raibert and others [12] implemented a pneumatic actuator into their one-leg hopping machine and demonstrated fantastic hopping motions. Later, many researchers [13]-[15] followed the same idea for hopping machines. 


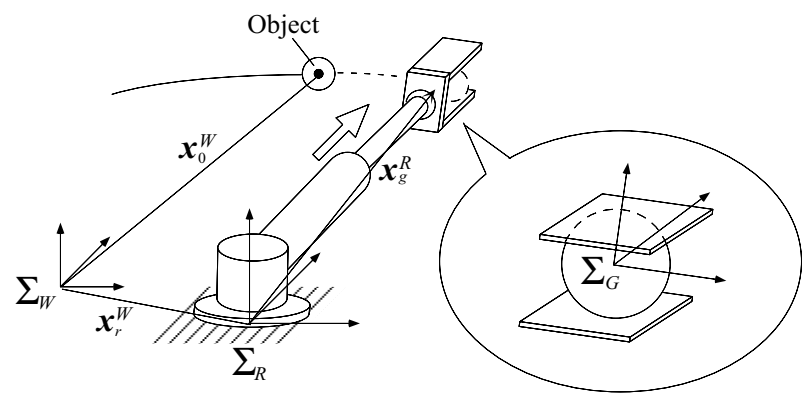

Fig. 3. Coordinate System of Robot and Object

While there are many works concerning with the mechanical design of robot manipulators, our goal is to design a capturing system with extremely high acceleration by introducing the accumulated energy and the AGCM newly introduced.

\section{Some Remarks for Capturing a Moving Object}

We first define $V\left(\boldsymbol{x}^{G}\right)$ as the vector function expressing the region enabling the robot to capture an object irrespective of orientation of object, where $\boldsymbol{x}^{G}$ denotes the vector whose origin is the geometrical center of gripper and $\sum_{G}$ is the gripper coordinate system. Mathematically, $V\left(\boldsymbol{x}^{G}\right)$ is given by,

$$
V\left(\boldsymbol{x}^{G}\right)=\left\{\begin{array}{l}
V\left(\boldsymbol{x}^{G}\right)>0: \text { Outside of } V\left(\boldsymbol{x}^{G}\right) \\
V\left(\boldsymbol{x}^{G}\right)=0: \text { Surface of } V\left(\boldsymbol{x}^{G}\right) \\
V\left(\boldsymbol{x}^{G}\right)<0: \text { Inside of } V\left(\boldsymbol{x}^{G}\right)
\end{array}\right.
$$

Generally, $V\left(\boldsymbol{x}^{G}\right)$ becomes small as the size of object increases and it finally results in zero with a particular size of object. Now, imagine that an object is moving in 3D space as shown in Fig.3, where $\sum_{W}, \sum_{R}, x_{o}^{W} \in R^{3}$, $\boldsymbol{x}_{r}^{W} \in R^{3}, \boldsymbol{x}_{g}^{R} \in R^{3}, R_{R}^{W} \in R^{3}$ and $R_{R}^{G} \in R^{3}$ are the world coordinate system, the robot coordinate system, the position vector of object in $\sum_{W}$, the position vector indicating the origin of $\sum_{R}$ in $\sum_{W}$, the position vector of the center of gripper in $\sum_{R}$, the rotational matrix from $\sum_{W}$ to $\sum_{R}$ and the rotational matrix from $\sum_{G}$ to $\sum_{R}$, respectively. The center of gripper is given by

$$
\boldsymbol{x}_{g}^{W}=\boldsymbol{x}_{r}^{W}+R_{R}^{W} \boldsymbol{x}_{g}^{R}
$$

where $\boldsymbol{x}_{g}^{W}$ denotes the position vector of the center of gripper in $\sum_{W}$. We assume that the gripper plate is massless and therefore it can move infinite velocity, while we suppose the inertia of arm is not negligible. As the necessary condition for catching, the gripper has to meet with a moving object. This condition is given as follows. 


\section{[Necessary Condition for Catching an Object]}

In order for a robot to capture a moving object, there should exist $\exists t$, such that it can satisfy

$$
\begin{aligned}
& V\left(\Delta \boldsymbol{x}^{G}\right) \leq 0 \\
& \Delta \boldsymbol{x}^{G}=\left(R_{G}^{R}\right)^{T}\left(R_{R}^{W}\right)^{T}\left\{\boldsymbol{x}_{g}^{W}(t)-\boldsymbol{x}_{o}^{W}(t)\right\}
\end{aligned}
$$

where $t$ and $T$ denote time and the transpose of matrix.

Without satisfying this condition, the robot can never meet a moving object within the capturing region $V\left(\boldsymbol{x}^{G}\right)$. However, note that this condition is not sufficient for stopping the object within the gripper. Because due to the inertia force, the object will slide even after the object is in contact with the inner surface of gripper. The sliding distance depends on the contact friction, the friction coefficient, and the velocity of object. Since we can not estimate the exact sliding distance in advance, the high speed capturing system should be designed so that the gripper may have an appropriate area for the gripper plate.

\section{Design Policy}

We can shoot out an arrow in the maximum acceleration of $150 \mathrm{G}$ in archery. To achieve such a high acceleration, there are two keys: The Small Mass of Arrow, greatly contributing to achieving a large acceleration under the same energy resource and The Bow Cord, accumulating a high energy in ready state. Learning such mechanical properties of archery, we design the robot by regarding both arrow and cord as robot arm and spring element, respectively. Additionally, we have to consider the gripper mechanism and the power transmission to it. Especially, from the viewpoint of quick response, we have to design the robot in such a way that we can remove the time lag between the arm and the gripper motions. To cope with this issue, we introduce the Arm/Gripper Coupling Mechanism (AGCM).

\subsection{Energy Accumulated Arm}

Fig.4 (a) shows the relationship between the archery and the basic part of the arm, where a spring is implemented at the base of the robot arm. In archery, the arrow is flew out in the air at the moment we release the cord. In order for the robot arm to avoid flying away, the base-wire is connected between the arm and the pulley fixed at the shaft of B-Motor (Base Motor) for adjusting the stop point of the arm. Fig.4 (b) shows more precise illustration of the base, where a permanent magnet and an electromagnet are connected at the end of arm and the base, respectively. Before staring the capturing motion, the B-Motor winds up the base-wire till the spring is fully compressed, as 




(a)

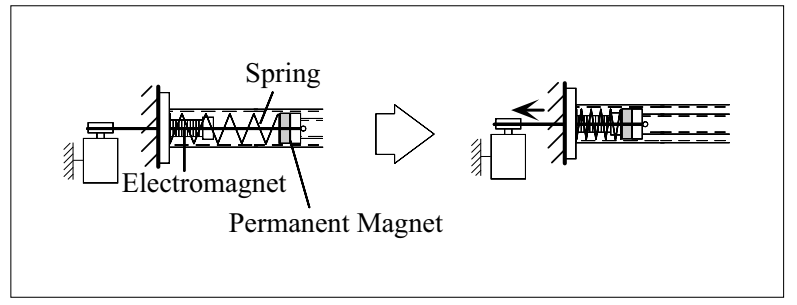

(b)

Fig. 4. Robot Mechanism Learnt by Archery

shown in Fig.4 (b). At the same time, the electromagnet is switched on, so that the arm is fully stopped. Then, the base-wire is released to the designated length, so that the arm can freely move up to the predetermined point. This is so called ready-phase. The moment the electromagnet is switched into the opposite polarity, the arm quickly starts in maximum acceleration and stops at the position in the predetermined length of the base-wire. Without any additional mechanism, the potential energy accumulated in the ready phase is completely lost and changed into heat, when the arm completely stops. As a result, we have to prepare another energy resource for operating the gripper, which will cause the increase of mass of moving part as well as the increase of the time lag between the arm and the gripper motions. These are, of course, not desirable from the viewpoint of both increase the acceleration and the decrease the response time. The Arm/Gripper Coupling Mechanism (AGCM) is the key for transmitting the energy from the spring to the arm, and from the arm to the gripper continuously, with a simple mechanism based on wire.

\subsection{Arm/Gripper Coupling Mechanism (AGCM)}

The gripper should have a small mass and a simple structure, so that it can quickly move. We utilize the so-called Soft Gripper proposed by Hirose [16], since it satisfies our requirements. It can operate by simply pulling and releasing the drive-wire. We implement a rotational spring in each joint, so that the returning motion can be produced automatically when the drive-wire is released. Now, a question is how to transmit the energy from the arm to the 


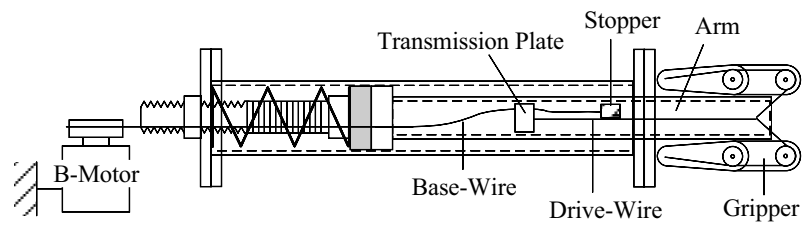

(a) Ready State of the Capturing System

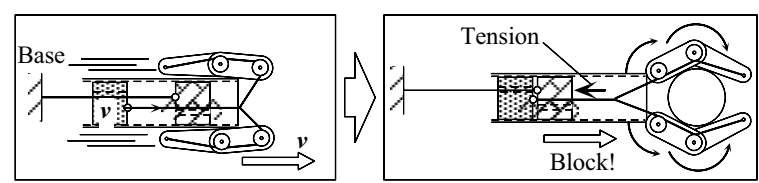

(b) Operation Mechanism

Fig. 5. Overview of AGCM

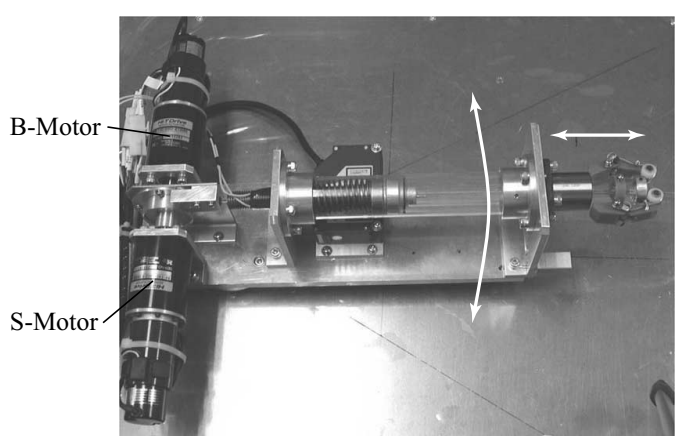

(a) Overview

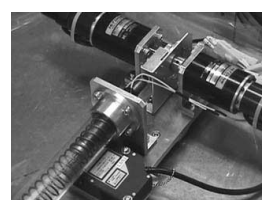

(b) Actuators

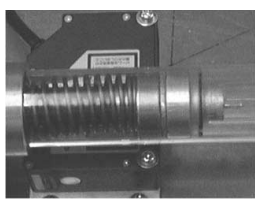

(c) Spring and Magnets

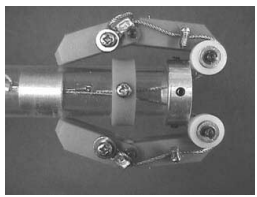

(d) Gripper

Fig. 6. Experimental System

gripper at the capturing point. This question can be replaced by how to pull the drive-wire at the predetermined point. For this question, we finally come to the simple mechanism as shown in Fig. 5 where the base wire is connected to the stopper through the hole of the transmission plate. In the ready state, the base-wire is loosen enough so that the arm can freely move until it is fully blocked by the stopper. The moment the polarity of electromagnet is changed, the arm is accelerated with the transmission plate. Although the inertia forces caused by both the transmission plate and the stopper pull the drive-wire in such a way that the gripper may be closed, we can choose appropriate 


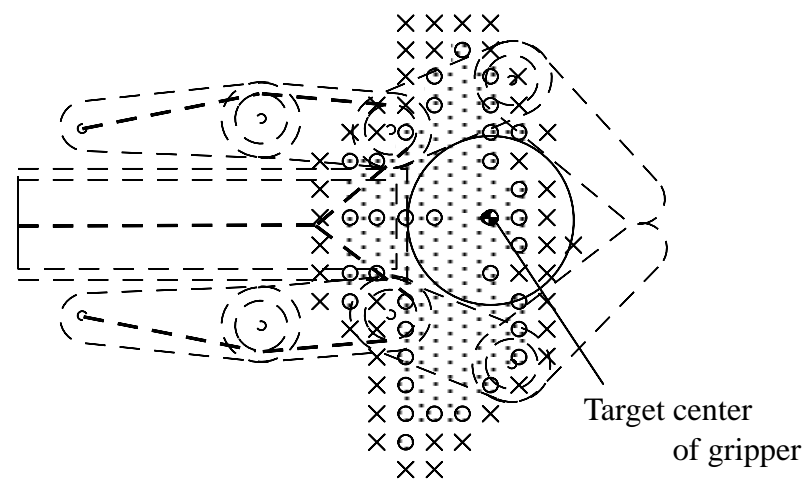

Fig. 7. $V^{\prime}\left(x^{G}\right)$

rotational springs of gripper, to overcome the moment coming from the inertia force. When the arm reaches the predetermined length as shown in Fig.5 (b), the transmission plate can no more move due to physically blocking by the stopper. At this moment, a large interaction force suddenly appears between the transmission plate and the stopper. As a result, a large tension appears in the drive-wire and makes the gripper close quickly without any time lag. By utilizing the AGCM, the kinetic energy of the arm is transmitted to the drivewire connected to the gripper through the transmission plate and the stopper. We would note that the compression of spring at the end of capturing motion produces the grasping force, while most of initial energy finally changes into the heat.

\section{Experiments}

\subsection{Experimental System}

Fig.6 shows the developed capturing system, where (a), (b), (c), and (d) are an overview of the system, the actuator for changing the tendon length, the spring and the magnets, and the gripper, respectively. There are three DC servo-motors: the S-Motor for compressing the spring, the B-Motor for adjusting the capturing point, and the R-Motor for rotating the whole system. The the developed capturing system can operate in two modes, the normal mode where we can use it as a normal planar robot by independently controlling both S- and B-Motors, and the high-speed mode where the S-Motor is released enough so that the stopping point can be determined by the BMotor only. The spring constant, the mass for both arm and grippers, and the absorption force between the electromagnet and the permanent magnet are $0.65 \mathrm{~N} / \mathrm{mm}, 100 \mathrm{~g}$, and roughly $71 \mathrm{~N}$, respectively. These specifications tell us the maximum acceleration of the developed capturing system is $73 \mathrm{G}$ under neglecting the effect on acceleration due to magnetic force. For detecting 


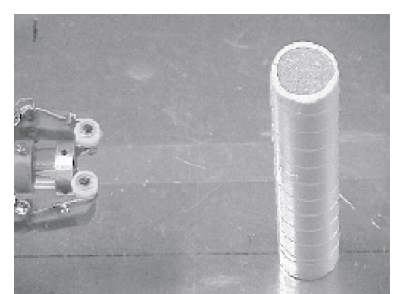

(a) $1 / 30[\mathrm{~s}]$

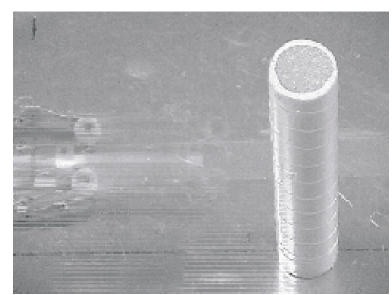

(c) $3 / 30[\mathrm{~s}]$



(e) $5 / 30[\mathrm{~s}]$

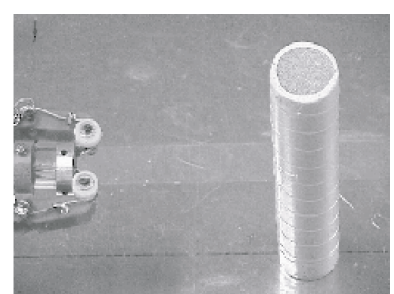

(b) $2 / 30[\mathrm{~s}]$

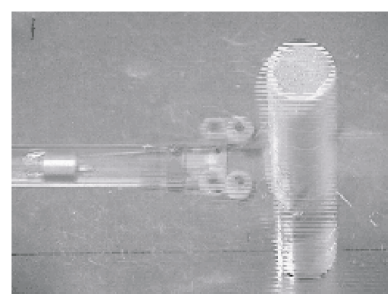

(d) $4 / 30[s]$

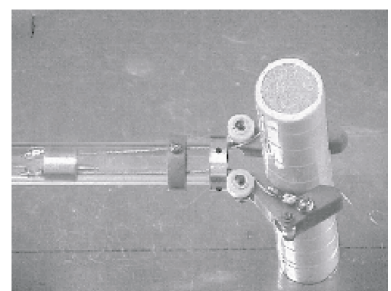

(f) $6 / 30[\mathrm{~s}]$

Fig. 8. High-speed Mode

the position of the target object, we implement a laser sensor (KEYENCE LB-1000) whose sampling time is $1 \mathrm{~ms}$. This sensor is installed at the base located just below the arm. While our goal is to utilize the $1 \mathrm{~ms}$-vision instead of the laser sensor, we temporally implement it for its easy treatment.

\subsection{Experimental Results}

We first choose the cylindrical object with the diameter of $30 \mathrm{~mm}$. We define the 2D capturing region $V^{\prime}\left(\boldsymbol{x}^{G}\right)$ where the target center of gripper denotes the reaching position of the center of gripper under no object. The robot can capture successfully the object when it is perpendicularly placed on the table and the center exists within $V^{\prime}\left(\boldsymbol{x}^{G}\right)$. Fig.7 shows the experimental results where the robot can capture the object when it is placed on the circle $(\bigcirc)$ points and the robot fails in capturing it when it is placed on the $\operatorname{cross}(\times)$ points. Fig. 8 shows a series of motion for capturing the cylindrical object placed on a table where (a), (b), (c), and (d) show the motion in every 1 frame $(1 / 30 \mathrm{sec})$ of the digital camera, respectively. Fig.9 shows both position and velocity with respect to time, where the position and the velocity 

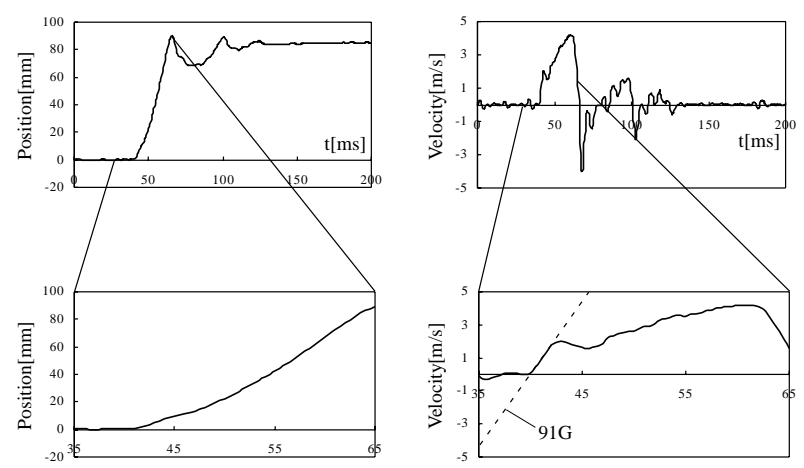

Fig. 9. High-speed Mode
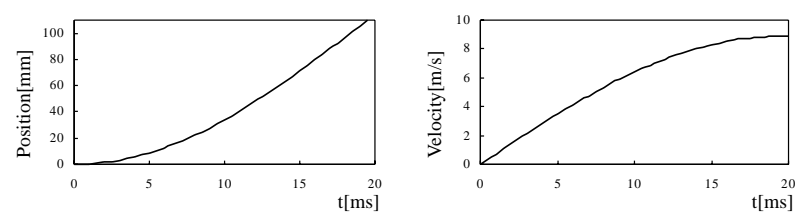

Fig. 10. Simulation

data are obtained by the laser sensor and the numerical differentiation of the position signal, respectively. This figure tells us that the time taken for the capturing motion is roughly $0.03 \mathrm{sec}$. We can observe the maximum acceleration of $91 \mathrm{G}$ in the initial phase and the average acceleration of roughly $22 \mathrm{G}$. An interesting observation is that the maximum acceleration observed by the experiment is $25 \%$ more than that predicted by an ideal model. This difference comes from that in the ideal model we neglected the effect on acceleration due to magnetic force immediately after changing the polarity. To validate this consideration, we show a simulation result in Fig.10 where the magnetic force is neglected. By comparing both Fig.9 and Fig.10, the effect on acceleration by magnetic force is clearly observed in the experiment, while it does not in the simulation. By using the the developed capturing system, we also challenged a difficult task where the robot catches a ball coming down from an appropriate height. A laser sensor mounted at the base can detect the distance up to the ball, when the ball passes the base level of the robot. The starting time is carefully controlled so that the gripper can capture the ball after bouncing at the table. Fig.11 shows an experimental result, where a ball dropped at the height of $20 \mathrm{~cm}$ is successfully captured by the developed system. To increase the success rate, there are two keys: (i) to exactly know the capturing time of the robot and (ii) to chase the object accurately. Regarding (i), a high-speed video may be effective for measuring the time till the gripper completely closes. The laser sensor can provide an accurate distance only when a laser hits the ball, while a vision system can always 

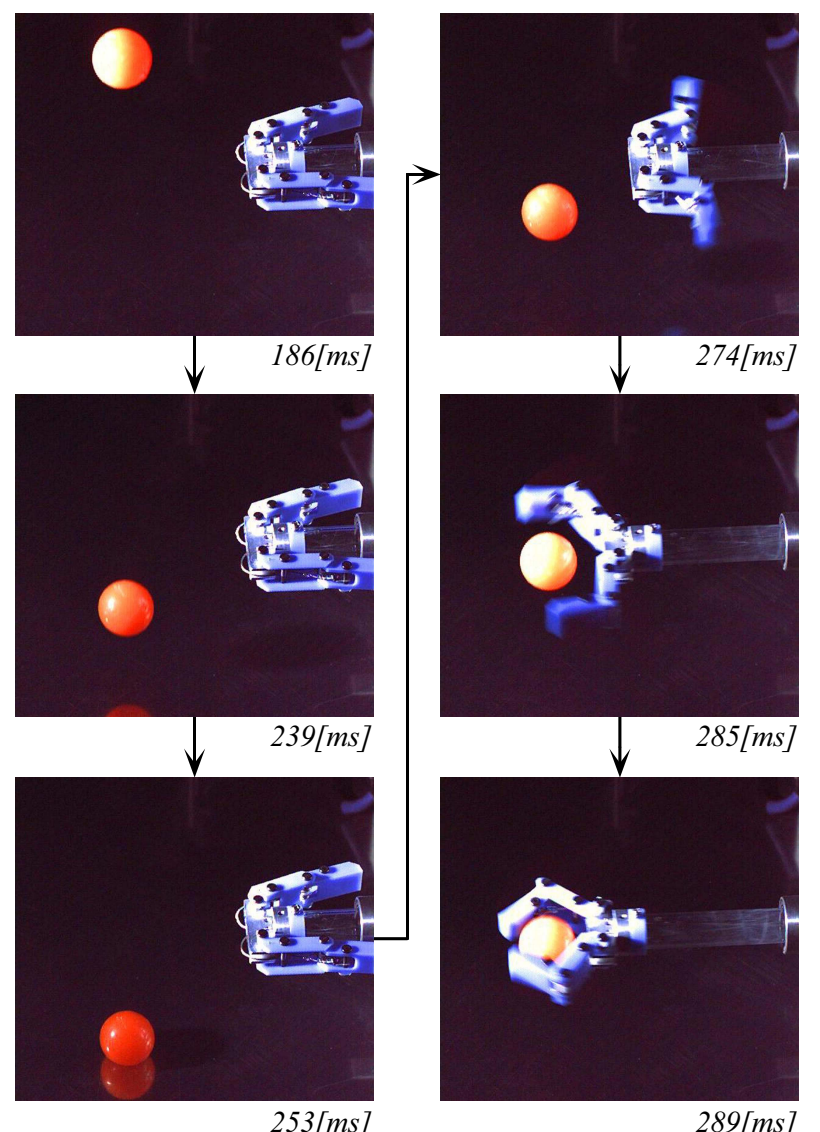

$253[\mathrm{~ms}]$

$289[\mathrm{~ms}]$

Fig. 11. An Experiment for Catching a Ball

chase it as far as it exists within the viewing range. Furthermore, when the laser spot hits near the boundary of ball, only partial reflected light comes back to the sensor, which leads to a large error for measuring the distance.

\section{Discussion}

Since every object has non-zero mass, we can not stop the object instantaneously. Depending upon the mass and the speed of object, the object will slide within the gripper until it finally comes to rest. Especially, as the capturing time decreases, and the initial kinetic energy of object increases, the sliding distance is no more negligible. For example, if the sliding length is more than the size of gripper, we can not stop the object within the gripper. Therefore, such a sliding distance will become an important index for determining not only the size of gripper but also the normal contact force. 


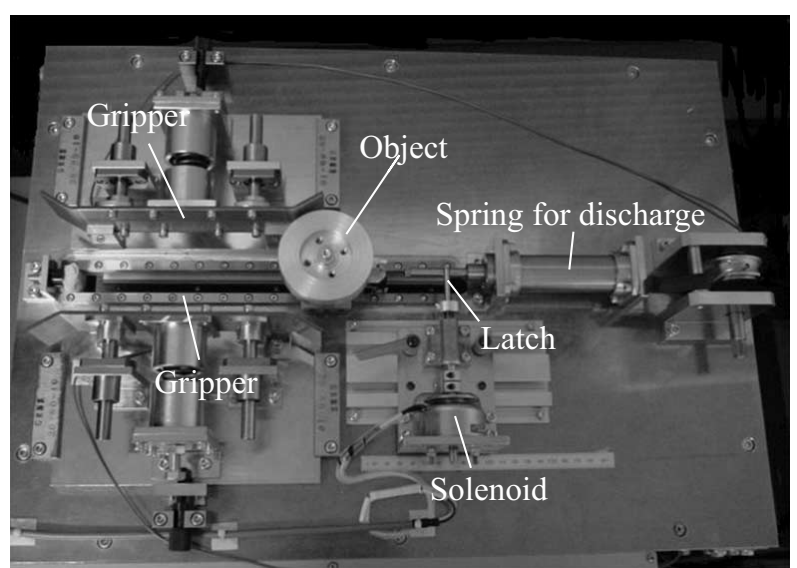

Fig. 12. Experimental system

In order to evaluate the sliding length under a given coustraint condition, we developed the test system with a gripper and an object, as shown in Fig.12, where the object can be launched by a spring. Both the translational and the rotating motions for the object can be given independently. The latch is released by PC command. The moment it is released, the object is pushed forward by the spring energy accumulated in advance. After the object makes contact with the gripper, it gradually slow down and finally comes to rest. We are interesting to know the sliding distance under the given kinetic energy and the normal contact force. Fig.13 shows the experimental results where the ration between the translational and the rotational velocities is changed under the same initial kinetic energy. Simulation result is obtained by utilizing the dynamic simulation software ADAMS. As a general tendency, the sliding distance becomes small as the rotational speed increases. The reason is that the rotational energy does not much contribute to increasing the sliding distance, while a longer sliding distance is needed for a larger translational kinetic energy. We would also note that there exists a non-negligible difference between the experimental and the simulation results. There are a couple of reasens for the difference. For example, simulation does not consider the energy loss due to inpact between the object and the gripper, and it is difficult to keep the condition where a constant normal force is applied to the object during contact. For more clear explanation, further experimental results will be needed for various experimental conditions.

\section{Conclusion}

We discussed the design of high-speed capturing system by using single energy resource. We proposed the Arm/Gripper Coupling Mechanism (AGCM), so that we can transmit the initially accumulated energy from the arm to the 


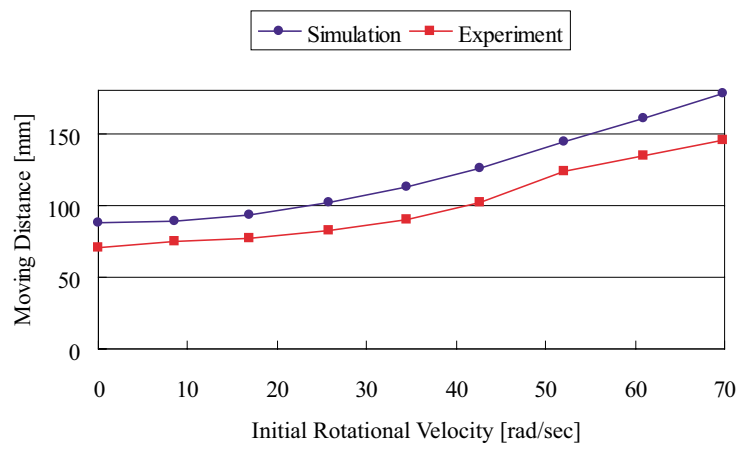

Fig. 13. Moving Distance

gripper. The experimental results by the developed robot showed the maximum acceleration of $91 \mathrm{G}$ corresponding to almost two times higher than the conventional world record, and the total capturing time of 0.03sec. Experiments on capturing a ball were shown.

This work was supported by the grant Creating a Brain Project of Core Research Evolutional Science and Technology (CREST), JST, Japan.

\section{References}

1. M. Ishikawa and M. Kaneko, (2001) Innovative Sensory-Motor Fusion Opens a New Robotic World, Note of IEEE ICRA. Tutorial

2. M. Kaneko, T. Tsuji, and M. Ishikawa, (2002) The Robot that can Capture a Moving Object in a Blink, Proc.of IEEE ICRA, pp 2001-2007

3. A. Namiki, Y. Nakabo, I. Ishii, and M. Ishikawa, (2000) 1-ms Sensory-Motor Fusion System, IEEE Trans.on Mechatronics, vol. 5, no. 3, pp 244-253

4. I. Ishii, and M. Ishikawa, (1999) Self Windowing for High Speed Vision, Proc.of IEEE ICRA, pp 1916-1921

5. J. P. Karidis, G. McVicker, J. P. Pawletko, L. C. Zai, M. Goldowsky, R. E. Brown, R. R. Comulada, (1992) The Hummingbird Minipositioner - Providing Three-Axis Motion At 50 G's With Low Reactions, IEEE Int.Conf.on $R \& A$, pp $685-692$

6. http://www.barretttechnology.com/robot/products/arm/armspfr.htm .

7. P. K. Allen, A. Timocenko, B. Yoshimi, and P. Michelman, (1993) Automated Tracking and Grasping of a Moving Object with a Robotic Hand-Eye System, IEEE Trans.on $R \& A$, vol. 9, no. 2, pp 152-165

8. W. T. Townsend, (1988) The Effect of Transmission Design on Force-Controlled Manipulator Performance, PhD Thesis, Department of Mechanical Engineering, $M I T$

9. W. Hong, J. E. Slotine, (1997) Experiments in Hand-Eye Coordination Using Active Vision, Lecture Notes in Control and Information Sciences, vol. 223, pp 130-139 
10. S. Kawamura, W. Choe, S. Tanaka, and S. R. Pandian, (1995) Development of an Ultrahigh Speed Robot FALCON Using Wire Drive System, Proc. of IEEE ICRA, pp 215-220

11. M. Uchiyama, T. Sadotomo, and K. Masukawa, (1996) Dynamic Control Experiment on a Parallel robot HEXA, J. of Robotics Society of Japan, vol. 14, no. 2, pp 297-304

12. M. H. Raibert, (1983) Dynamically Stability and Resonance in a Legged Hopping Machine, IFToMM Proc. of Symp. on Theory and Practice of Robots and Manipulators, pp 352-367

13. M. H. Raibert, (1990) Trotting, Pacing, and Bounding by a Quadruped Robot, J. of Biomechanics, 23, pp 79-98

14. M. D. Berkemeier and K. V. Desai, (1996) Design of a Robot Leg with Elastic Energy Storage, Proc. of IEEE ICRA, pp 213-218

15. B. V. Chapnik, G. R. Heppler, and J. D. Aplevich, (1991) Modeling Impact on a One Link Flexible Robotic Arm, IEEE Trans.on R\&A, vol. 7, no. 4, pp 479-488

16. S. Hirose and Y. Umetani, (1983) Soft Gripper, Proc. of ISIR, pp 112-127 